\title{
A coil-globule transition of a semiflexible polymer driven by the addition of spherical particles
}

\author{
Richard P. Sear \\ Department of Chemistry and Biochemistry \\ University of California, Los Angeles \\ Los Angeles, California 90024, U.S.A. \\ email: sear@chem.ucla.edu
}

February 13, 2018

\begin{abstract}
The phase behaviour of a single large semiflexible polymer immersed in a suspension of spherical particles is studied. All interactions are simple excluded volume interactions and the diameter of the spherical particles is an order of magnitude larger than the diameter of the polymer. The spherical particles induce a quite long ranged depletion attraction between the segments of the polymer and this induces a continuous coil-globule transition in the polymer. This behaviour gives an indication of the condensing effect of macromolecular crowding on DNA.
\end{abstract}

\section{Introduction}

Phase separation and partitioning driven by excluded volume interactions have been well studied theoretically [1] 8], with the inspiration coming from experiments both on synthetic colloidal systems [2, 3, 9] and on biologically derived systems [10,11]. When excluded volume effects are dominant the properties of a mixture are determined solely by the sizes and shapes of its components. For example, mixtures of long narrow rod-like particles and spheres have been shown to demix solely because of these differences in size and shape [5, 7]. The rod-like particles could be a minimal model of a semiflexible polymer or of a micelle, and the spheres could be small colloidal particles or even compact protein molecules. But if the semiflexible polymer is very long then even a single, isolated molecule can undergo phase transitions 12 16], because it is then large enough, has enough degrees of freedom, to be treated as a thermodynamic system [12]. Here we study such a polymer, mixed with spheres, and see if the presence of the spheres can induce a phase transition. We find that they can. When the concentration of spheres exceeds a critical value the polymer molecule contracts and expels the spherical particles. In effect the polymer molecule and the spheres are demixing. The mixture of many short rod-like polymers and spheres demixed to form a phase with a high density of rod-like polymers but a low density of spheres (coexisting with one with high sphere density and low rod density) and here the polymer contracts to form a dense globule with a high density of polymer but a low density of spheres. This dense phase of a polymer is referred to as the globular phase [12] 16] and the contraction of the polymer is the coil-globule transition.

In the work presented here, we will draw on existing theories for the demixing of spheres and semiflexible polymers [7] and for the coil-globule transition [14, 15]. The theory for the mixture of spheres and semiflexible polymers [7] is a straightforward virial expansion of the Helmholtz free energy, truncated after the second virial coefficient terms. In the free energy expressions of Ref. [7] the two components of the mixture were treated symmetrically. While this is of course perfectly valid, here we want to use the analogy between demixing and the vapour-liquid phase separation of a pure substance 11 3, 177. To do this we will Legendre transform [18] the Helmholtz free energy into a semi-grand potential [17, 19]. This semi-grand potential is then a function of the density of the polymer and the chemical potential of the spheres. A chemical 
potential, like the temperature, is uniform throughout any system at equilibrium; it is a field variable not a density. So, the semi-grand potential has the same form as the Helmholtz free energy of a polymer which interacts via interactions which are not solely excluded volume and so depend on temperature. Both depend on the density of polymer and on a field variable: temperature in the case of a polymer with soft interactions, and the chemical potential of the spheres for the polymer mixed with spheres. In particular, both thermodynamic functions can be expanded as a virial series in the density of polymer with coefficients which depend on temperature/chemical potential of the spheres. Just as reducing the temperature of a polymer molecule with attractive interactions can make some of its virial coefficients negative, increasing the chemical potential of spheres drives some of the virial coefficients of the semi-grand potential of the mixture of polymer and spheres negative. In both cases the effect is the same: the polymer contracts from the coil state to the globular state. The spheres have in effect induced an attraction, often called a depletion attraction, between the segments of the polymer molecule.

The interactions between the segments of a polymer molecule determines its state. Note that we always consider a single, isolated polymer molecule. If the interactions between the polymer segments are repulsive, the good solvent regime, then the polymer exists as a swollen coil [12]. The radius of gyration of the polymer, a measure of its size, scales with the number of segments $N$ as $N^{3 / 5}$ (actually the exponent is slightly less than $3 / 5[16])$. So, the volume occupied by the polymer molecule scales as $N^{9 / 5}$. The exponent is greater than one and so the average density of segments inside this volume tends to 0 as $N$ tends to infinity. However, if the interactions between the segments of the polymer are sufficiently attractive, the poor solvent regime, the polymer exists in a condensed state, the globular state [12, 14 16]. There the radius of gyration of the polymer scales as $N^{1 / 3}$ and so as $N$ tends to infinity the average density inside the polymer remains non-zero. The crossover from the radius of gyration scaling as $N^{3 / 5}$ to $N^{1 / 3}$ marks the coil-globule transition.

Motivation for studying the current model mixture is provided by an interest in the phase behaviour of long DNA double helices. The DNA double helix is a semiflexible polymer, its persistence length [20] is $\sim 50 \mathrm{~nm}$ [21] which is 25 times its diameter of $\sim 2 \mathrm{~nm}$. Our semiflexible polymer with only excluded volume interactions is a crude model of DNA in a good solvent [20,21]. Our model mixture is then a crude but not unreasonable model of a long DNA molecule in a suspension of spherical particles, in the absence of any specific DNA-spherical-particle attraction. Thus, our results imply that DNA can be condensed using colloidal spheres. As far as we are aware this has not been attempted. However, there are a number of experimental techniques for condensing DNA, such as altering the solvent, adding polyvalent salts etc., see Refs. 22, 23 and references therein. However, all these techniques produce a sudden collapse of the DNA to a dense state in which the separation between adjacent parts of the DNA is only a few nm and the DNA has hexagonal order. The collapse induced by the colloidal spheres is continuous and and it is then possible to prepare a low density isotropic globule of DNA. As far as we are aware this is the only way of preparing a low density globule of a semiflexible polymer such as DNA. Our system may also be useful as a very crude model of the effect of 'macromolecular crowding' on DNA actually in cells, see Refs. 10, 11, 24] and references therein.

\section{Model}

The polymer is modeled by a homogeneous cylindrical elastic filament 20]. The filament follows a continuous curve in space, see Fig. 1. The filament bends and flexes during thermal motion but it has a certain amount of rigidity, measured by its persistence length $P[20$. A piece of polymer shorter than the persistence length only bends by a small amount due to thermal motion, it behaves almost like a rigid rod. The polymer has a hard core of diameter $D$, that is the centreline of the polymer cannot approach within $D$ of itself. The polymer is $N$ persistence lengths long; in our calculations we will always consider the limit of $N \rightarrow \infty$.

The colloidal spheres are modeled by hard spheres with a diameter $D_{s}$. The polymer-sphere interaction is also an excluded-volume interaction. The centre of a sphere cannot approach within $\left(D+D_{s}\right) / 2$ of the centreline of the polymer. 


\section{Theory}

We only consider explicitly the globular state of a single isolated polymer molecule in the $N \rightarrow \infty$ limit. We also neglect any variation in the density of polymer segments in the globule; the volume approximation of Lifshitz and coworkers 14, 15. Then the globule is simply a bulk phase of volume $V$ in which the $N$ polymer segments are distributed with a uniform density $\rho=N / V$. Far from the coil-globule transition and for large $N$ this is reasonable, then the globule is expected to resemble a drop of liquid - the density is uniform except for a narrow interfacial region at the surface of the globule. It should be borne in mind that the assumptions behind our free energy break down at the transition itself. They provide an estimate of what density of colloidal particles is required to induce a coil-globule transition of the polymer but cannot say anything about the critical behaviour at the transition. For a detailed study of the region of the transition, see Ref. [25] and references therein.

The starting point is a virial expansion of the Helmholtz free energy $A_{g}$, of a globule, in the $N \rightarrow \infty$ limit. This free energy has had the free energy of the polymer in the ideal coil state subtracted off. The volume $V$ enclosed by the globule includes solvent, which we do not treat explicitly, and colloidal spheres. These spheres are at a density $\rho_{s}$ which is uniform within the globule. Then [14, 20]

$$
\frac{\beta A_{g}}{V}=\rho^{2} B_{2}+\rho_{s}\left[\ln \rho_{s}-1\right]+\rho_{s}^{2} B_{2}^{s s}+\rho \rho_{s} B_{2}^{p s},
$$

where $\beta=1 /(k T)$, for $T$ the temperature and $k$ Boltzmann's constant. The virial coefficients $B_{2}, B_{2}^{s s}$ and $B_{2}^{p s}$ are the second virial coefficients of the polymer-polymer, the sphere-sphere and the polymer-sphere interactions, respectively. We have neglected the contribution of the momentum degrees of freedom as they do not affect the phase behaviour. It is this which has caused the argument of the logarithm in Eq. (1) to have dimensions of inverse volume. In the absence of colloid $A_{g}$ is just equal to the first term on the right hand side of Eq. (11). This term gives the increase in free energy with density due to the excluded volume interactions. The entropy cost in compressing a coil into a globule with a finite density $\rho$ is not of order $N$ and so is not included in Eq. (11). The virial coefficients are given by

$$
\begin{aligned}
B_{2} & =\frac{\pi}{4} P^{2} D \\
B_{2}^{s s} & =\frac{2 \pi}{3} D_{s}^{3} \\
B_{2}^{p s} & =\frac{\pi}{4} P\left(D+D_{s}\right)^{2} .
\end{aligned}
$$

$B_{2}^{s s}$ is the second virial coefficient of hard spheres of diameter $D_{s}$. The above expression for $B_{2}^{p s}$ is the volume a rigid cylinder of diameter $D$ excludes to a sphere of diameter $D_{s}$. Equation (2) for $B_{2}^{p s}$ therefore neglects the curvature of the polymer, but so long as $P>D_{s}$ the polymer curves gently on a length scale of $D_{s}$ and this approximation is a mild one. The second virial coefficient of the polymer-polymer interactions $B_{2}$ is obtained by splitting the polymer into segments of length less than $P$ but much larger than $D$ and then assuming that these interact as rigid rods [20]. Consider a polymer of length $L$, we split it up into $L / l$ segments of length $l$. The excluded volume of two cylinders of length $l$ is $(\pi / 4) l^{2} D$ if $l \gg D$. So, the volume excluded to one segment by the others is $(\pi / 4) L l D$, and this times the number of segments $L / l$ gives the total excluded volume, $(\pi / 4) L^{2} D$. Dividing this total excluded volume by $V$ and realising that $L / P=N$ we see that we have obtained the first term in Eq. (11).

The volume $V$ occupied by the globule is within a much larger volume of colloidal suspension which acts as a reservoir of colloid, thus fixing its chemical potential. The density of the colloidal spheres is different inside and outside of a globule, thus it is more convenient to work with not the density of the spheres but their chemical potential, which is of course always uniform. The coil-globule transition is then brought about by increasing the chemical potential of the spheres. (This is completely analogous to inducing a coil-globule transition by reducing the temperature.) The chemical potential $\beta \mu_{s}$ is equal to the derivative of the free energy of Eq. (11) with respect to $\rho_{s}$

$$
\beta \mu_{s}=\ln \rho_{s}+2 \rho_{s} B_{2}^{s s}+\rho B_{2}^{p s} .
$$


Under conditions of fixed $\rho$ and $\mu_{s}$ the correct thermodynamic potential is not the Helmholtz free energy but a semigrand potential $\Omega_{g}$ defined by [18]

$$
\frac{\Omega_{g}}{V}=\frac{A_{g}}{V}-\rho_{s} \mu_{s}
$$

Using Eqs. (11) and (3) this becomes

$$
\frac{\beta \Omega_{g}}{V}=\rho^{2} B_{2}-\rho_{s}-\rho_{s}^{2} B_{2}^{s s} .
$$

We now go over to reduced units. Two reduced densities are defined: $\zeta=\rho B_{2}$ and $\phi_{s}=\rho_{s} B_{2} / 4$. For semiflexible chains with $P \gg D$ the isotropic-nematic transition occurs between an isotropic phase with $\zeta=5.12$ and a nematic phase with $\zeta=5.51$ [20]. $\phi_{s}$ is the volume fraction of colloid. Hard spheres solidify at a volume fraction close to a half. We have given the reduced densities at which semiflexible polymers and spheres order as they give a good idea of the densities at which interactions are significant. For densities much less that those given for the transitions the polymer/fluid of spheres is close to ideal. Then in reduced units, Eq. (5) is

$$
\frac{\beta \Omega_{g}}{N}=\zeta-\frac{3}{2} \frac{\phi_{s}}{\zeta}\left(1+4 \phi_{s}\right)
$$

and Eq. (3) is

$$
\ln z_{s}=\ln \phi_{s}+8 \phi_{s}+\left(\frac{D}{P}\right)\left(1+\frac{D_{s}}{D}\right) \zeta
$$

where $z_{s}=\exp \left(\beta \mu_{s}\right) B_{2}^{s s} / 4$ is a reduced activity of the colloid. The volume fraction $\phi_{s}$ of an ideal gas of colloidal spheres in the absence of polymer is equal to $z_{s}$, in the presence of excluded volume interactions $\phi_{s}<z_{s}$. Note that Eq. (6) expresses $\Omega_{g}$ as a function of $\rho_{s}$ not $z_{s}$. To calculate $\Omega_{g}$ as a function of $z_{s}$ we have to solve Eq. (ब) for $\phi_{s}$ at the specified value of $z_{s}$. This can be done numerically. The pressure $p$ of the globule can be obtained by taking the derivative of $\Omega_{g}$, Eq. (6), with respect to $\zeta$.

\section{Results and Discussion}

A mixture is specified by the values of the three length scales: $D, P$ and $D_{s}$. All interactions are athermal and so the only energy scale is $k T$. Phase behaviour is solely determined by dimensionless ratios and the only dimensionless ratios that can be defined are then those between lengths. The mixture's phase behaviour is determined by two dimensionless ratios of lengths, we choose $P / D$ and $D_{s} / D$. The persistence length of DNA is around $50 \mathrm{~nm}$ or 25 times its diameter of $2 \mathrm{~nm}[21]$. So, we set $P / D=25$. The ratio $D_{s} / D$ is set equal to 15 for our calculations. This value is chosen as we estimate (see below) that for values of $D_{s} / D$ of order 10 the continuous coil-globule transition is not preempted by a collapse to a dense hexagonal globule. We will return to this point when we discuss our results.

At equilibrium the pressure is uniform which means that it must be the same inside the globule as in the surrounding colloidal suspension. A stable globule is then only possible if its pressure equals the pressure of the surrounding suspension, which is given by the $\zeta=0$ limit of the pressure derived from $\Omega_{g}$. Local stability also requires that at that value of $\zeta$ the pressure is an increasing function of $\zeta$.

In Fig. (2) we have plotted pressure-density plots at two values of $z_{s}, 0.5$ and 1 . At the smaller value of $z_{s}$ the pressure is a monotonically increasing function of the density of the polymer and so no phase with nonzero $\zeta$, i.e., no globular phase, is stable. At $z_{s}=0.5$ the polymer exists as a coil. However, for $z_{s}=1$ the pressure first decreases, goes through a minimum and then increases. So, here the coil state is unstable and the polymer exists at a density given by the condition that its pressure equals the pressure at $\zeta=0$. Note that $z_{s}$ can easily be converted into the density of colloid in the suspension outside the globule using

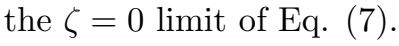

As the chemical potential of the spheres is increased the slope of the pressure versus $\zeta$ curve at $\zeta=0$ goes continuously to zero and then becomes negative. This corresponds to a continuous coil-globule transition. This is seen in Fig. 3. where we show the density of the globule as a function of $z_{s}$. As the transition is approached from above, i.e., high values of $z_{S}$, the density of the globule goes continuously to zero. The 
slope of the pressure curve at $\zeta=0$ is given by the coefficient of the term linear in $\zeta$ in the $\Omega_{g}$ - the second virial coefficient term in $\Omega_{g}$. The transition is at the point when this second virial coefficient equals 0 . There the third virial coefficient is positive and so the $\Omega_{g}$ of Eq. (6) has the same form as the free energy studied by de Gennes [12, 26].

We have found a continuous coil-globule transition for our semiflexible polymer. However, experiments on DNA 22, 23] show a first order transition; as does a theory for polymers of long rigid segments with short ranged attractions [28]. The reason for the difference is that the colloidal spheres induce a depletion attraction with a range $\sim D_{s}$. This is not much shorter than the polymer's persistence length, in contrast to the attraction in both the DNA in the experiments [22, 23] and the model of Ref. [28]. When the range of the attraction is much less than the persistence length, then the higher order virial coefficients are already negative when the second virial coefficient becomes negative [27,28]. The free energy is then not of the form considered by de Gennes and others [12 16, 26], and the continuous transition is preempted by a first order transition to a dense globule with at least nematic ordering. Thus, we predict that if the coil-globule transition of DNA is induced not by altering the quality of the solvent or adding polyvalent ions but by mixing in a colloidal suspension the transition will be continuous not first order.

Equation (6) for $\Omega_{g}$ is only valid when the polymer is in the isotropic phase. We now check that the transition predicted with this potential is not preempted by a transition to a dense ordered phase. This is easy to do as the isotropic-nematic transition of (pure) semiflexible chains is at a reduced pressure $p^{\prime}=\beta p B_{2} \simeq 26$ [20], much higher than the pressure of the transition we have found, see Fig. (2). There is no possibility of a dense nematic or hexagonal globule forming at this density of the colloid; the pressure inside any such globule would be much higher than the pressure of the colloidal suspension outside. Note that this conclusion relies on the spheres being large, i.e., on the ratio $D_{s} / D$ being large. At fixed volume fraction of the colloidal suspension, its pressure varies as $D_{s}^{-3}$. For values of $D_{s}$ of the order of $D$ the situation is very different. A suspension of spheres of this size can easily be at a pressure equal to the pressure of a dense (pure) nematic or hexagonal phase of the polymer. In addition, the overlap of the excluded volumes of two polymer segments only occurs when the two segments are close, within $D_{s} \sim D$ of each other. By excluded volumes we mean the volumes of space excluded by the polymer segments to the spheres [1]. Thus the attraction is now short ranged, its range is much less than $P$ and we expect a first order transition, as in Ref. [28].

Finally, in Fig. (4) we show the colloid density inside and outside the globule, as a function of $z_{s}$. We see that for the values of the parameters $P / D$ and $D_{s} / D$ that we have taken, a volume fraction of colloid a little more than 0.15 is required to induce the coil-globule transition. The colloid density outside the globule is just that of a fluid of hard spheres at that value of $z_{s}$, within the second virial coefficient approximation. It therefore increases as $z_{s}$ increases. The colloid density inside the globule, however, decreases as $z_{s}$ increases, due to the increasing polymer density of the globule.

\section{Conclusion}

We have studied a system of a large, isolated semiflexible polymer molecule in a suspension of spherical particles of diameter an order of magnitude larger than the diameter of the polymer but much much less than the radius of gyration of the polymer. The solvent for the polymer was good so we found that at low densities of the spheres the polymer was a swollen coil. However, as the density of spheres was increased beyond a certain point the polymer underwent a coil-globule transition. The polymer molecule and the spheres 'demixed': the polymer contracted to form a dense phase (Fig. 3) partially expelling the spheres (Fig. 4). The driving force for the coil-globule transition is the same as that for the demixing into two bulk phases of long rod-like particles and spherical particles [7]: the excluded volume interaction between the spheres and rods is large and this greatly reduces the volume available to the particles. The reduction in volume greatly reduces the translational and rotational entropy in phases which have high densities of both rods and spheres, favouring demixing into two phases, each with a high density of one component but a low density of the other.

The coil-globule transition we have found is continuous. Although flexible polymers such as polystyrene 15, 29] show a continuous coil-globule transition, for DNA the transition is discontinuous [22, 23]. The DNA coil suddenly collapses to form a dense globule with hexagonal ordering. In the experiments which showed the discontinuous collapse of DNA, the transition was brought about by some combination of polyvalent 
ions, alcohols and small polymer molecules, see Refs. [22, 23] and references therein. A natural assumption to make is that the collapse to a dense globule is then brought about by a short ranged attraction, i.e., two DNA helices only attract each other when they are a few $\mathrm{nm}$ apart. As discussed by the author in Refs. [27, 28], the dramatic collapse to a dense ordered phase is then not surprising, see also Refs [30 32]. The depletion attraction between polymer segments due to the presence of the spheres is long ranged, the range is not of order $D$ but of order $D_{s}$ which is an order of magnitude larger. It is this difference in range which is changing the coil-globule transition from discontinuous to continuous. Thus adding colloidal particles of size $\sim 20 \mathrm{~nm}$ or larger to DNA may produce a continuous collapse of the DNA. Such a continuous collapse has not yet been seen, as far as the author is aware.

\section{Acknowledgments}

It is a pleasure to thank S. Fraden for bringing references [10,11] to my attention. This work was started as a Royal Society Fellow at the FOM institute AMOLF in Amsterdam. I would like to thank The Royal Society for the award of a fellowship and AMOLF for its hospitality. 


\section{References}

[1] A. Vrij, Pure Appl. Chem. 48, 471 (1976).

[2] W. C. K. Poon and P. N. Pusey, Observation, Prediction and Simulation in Complex Fluids, M. Baus, L. F. Rull and J. P. Ryckaert, editors (Kluwer, Dordrecht, 1995).

[3] H. N. W. Lekkerkerker, P. Buining, J. Buitenhuis, G. J. Vroege and A. Stroobants, Observation, Prediction and Simulation in Complex Fluids, M. Baus, L. F. Rull and J. P. Ryckaert, editors (Kluwer, Dordrecht, 1995).

[4] M. Dijkstra and D. Frenkel, J. Chem. Phys. 101, 3179 (1995).

[5] P. Bolhuis and D. Frenkel, J. Chem. Phys. 101, 9869 (1994).

[6] R. van Roij and B. M. Mulder, Phys. Rev. E 54, 6430 (1996).

[7] R. P. Sear and B. M. Mulder, J. Phys. Chem. B 101, 4839 (1997).

[8] P. G. Bolhuis, A. Stroobants, D. Frenkel and H. N. W. Lekkerkerker, J. Chem. Phys. 107, 1551 (1997).

[9] A. D. Dinsmore, A. G. Yodh and D. J. Pine, Nature 383, 239 (1996).

[10] H. Walter and D. E. Brooks, FEBS Lett. 361, 135 (1995).

[11] A. P. Minton, Biophys. Chem. 57, 1 (1995).

[12] P. G. De Gennes, Scaling Concepts in Polymer Physics (Cornell University Press, Ithaca, 1979).

[13] C. Williams, F. Brochard and H. L. Frisch, Ann. Rev. Phys. Chem. 32, 433 (1981).

[14] I. M. Lifshitz, A. Yu. Grosberg and A. R. Khokhlov, Rev. Mod. Phys. 50, 683 (1978).

[15] A. Yu. Grosberg and D. V. Kuznetsov, Macromolecules 25, 1970 (1992); ibid 25, 1980 (1992); ibid 25, 1991 (1992); ibid 25, 1996 (1992).

[16] J. des Cloizeaux and G. Jannink, Polymers in Solution (Oxford University Press, Oxford, 1990).

[17] H. N. W. Lekkerkerker, W. C. K. Poon, P. N. Pusey, A. Stroobants and P. B. Warren, Europhys. Lett. 20, 559 (1992).

[18] D. Chandler, Introduction to Modern Statistical Mechanics (Oxford University Press, Nwe York, 1987).

[19] R. P. Sear and G. Jackson, J. Chem. Phys. 103, 8684 (1995).

[20] G. J. Vroege and H. N. W. Lekkerkerker, Rep. Prog. Phys. 55, 1241 (1992).

[21] K. Merchant and R. L. Rill, Macromolecules 27, 2365 (1994).

[22] M. Ueda and K. Yoshikawa, Phys. Rev. Lett. 77, 2133 (1996).

[23] K. Yoshikawa and Y. Matsuzawa, J. Am. Chem. Soc. 118, 929 (1996).

[24] L. D. Murphy and S. B. Zimmerman, Biophys. Chem. 57, 71 (1995).

[25] P. Grassberger and R. Hegger, J. Chem. Phys. 102, 6881 (1995).

[26] P. G. de Gennes, J. Phys. (France) 36, L-55 (1975).

[27] R. P. Sear, Phys. Rev. E 55, 5820 (1997).

[28] R. P. Sear, J. Chem. Phys., in press (cond-mat/9707283 at http://xxx.lanl.gov/).

[29] S.-T. Sun, I. Nishio, G. Swislow and T. Tanaka, J. Chem. Phys. 73, 5971 (1980). 
[30] A. Yu. Grosberg and A. R. Khokhlov, Adv. Polymer Sci. 41, 53 (1981).

[31] J. P. K. Doye, R. P. Sear and D. Frenkel, cond-mat 9709133 at http://xxx.lanl.gov/.

[32] U. Bastolla and P. Grassberger, cond-mat 9705178 at http://xxx.lanl.gov/.

\section{Figure captions}

Fig. 1 A schematic of our semiflexible polymer (the solid curve) immersed in a suspension of spherical particles (the shaded circles).

Fig. 2 Plots of the reduced pressure $p^{\prime}=\beta p B_{2}$ of a globule as a function of $\zeta$, at constant $z_{s}$. The solid curve (plotted on the left hand pressure scale) is for $z_{s}=0.5$, and the dashed curve (plotted on the right hand scale) is for $z_{s}=1$.

Fig. 3 The density of the globule $\zeta$ as a function of the colloid's activity $z_{s}$. The density goes to 0 at the coil-globule transition, which is at $z_{s}=0.515$.

Fig. 4 The density of the colloidal spheres $\phi_{s}$ outside (solid curve) and inside (dashed curve) as a function of the colloid's activity $z_{s}$. 


$$
8
$$




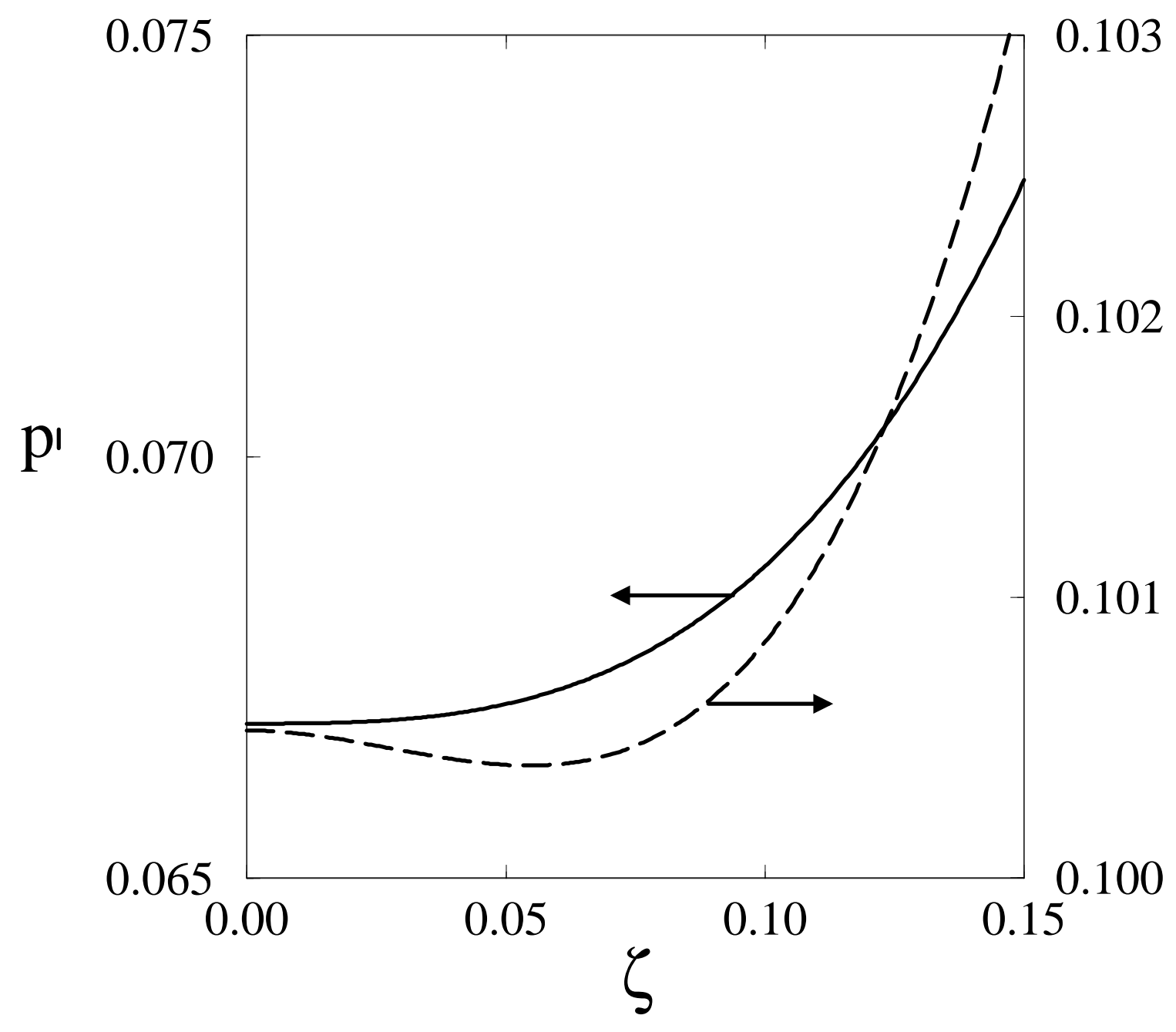




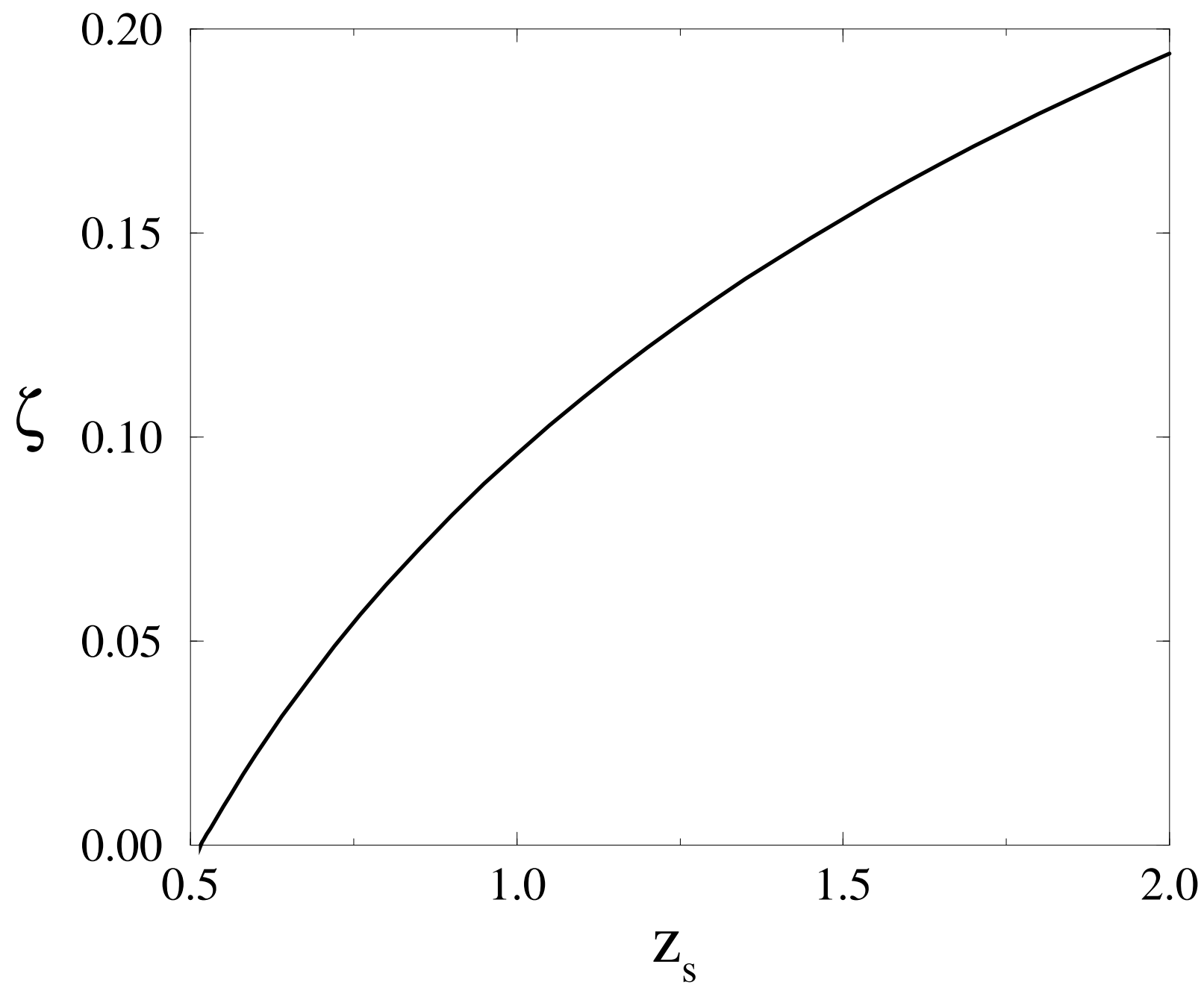




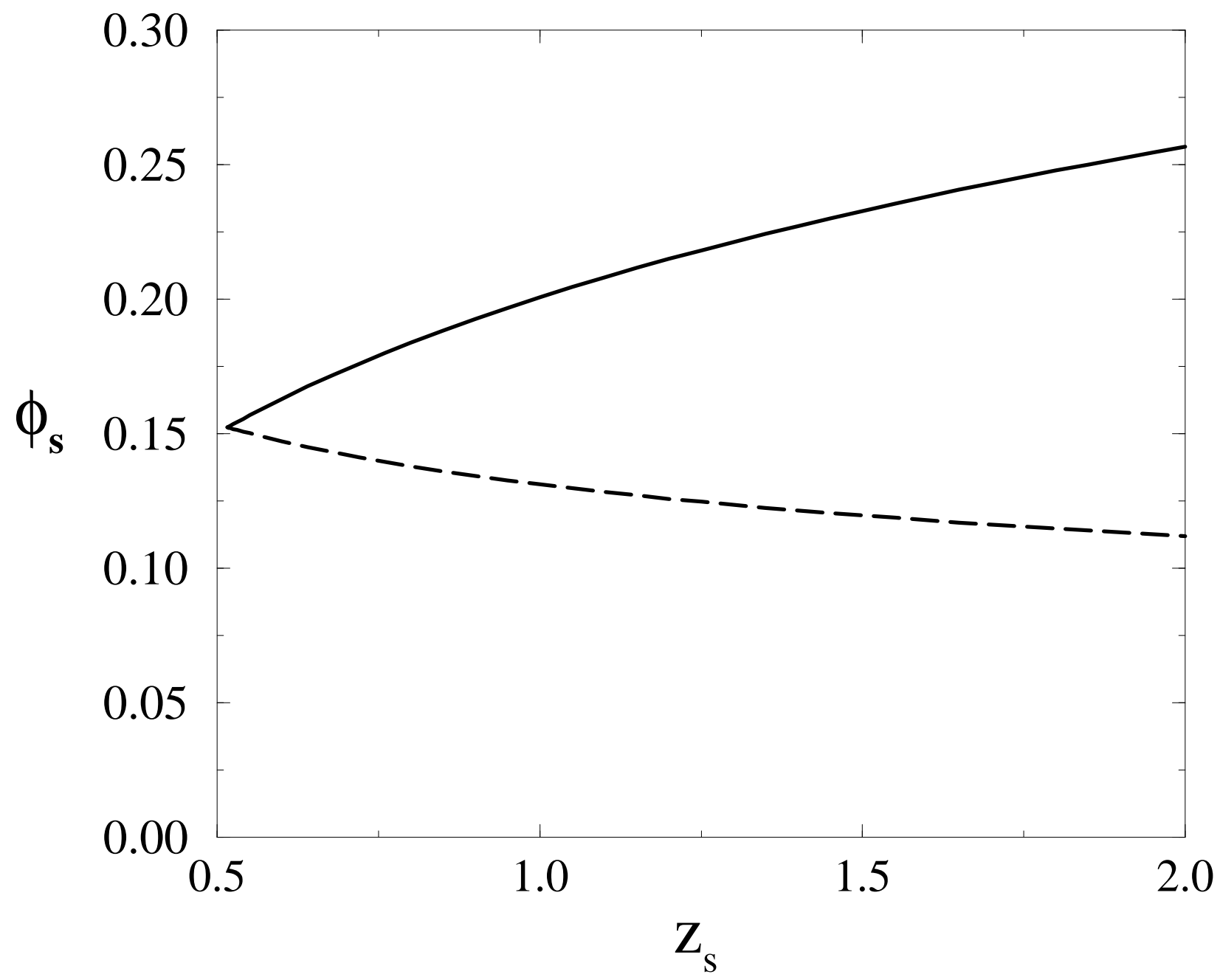

